\title{
Article \\ Implications of the Conformal Higgs Model
}

\author{
R. K. Nesbet
}

IBM Almaden Research Center, 650 Harry Road, San Jose, CA 95120-6099, USA; rkn@earthlink.net

\begin{abstract}
The postulate of universal local Weyl scaling (conformal) symmetry modifies both general relativity and the Higgs scalar field model. The conformal Higgs model (CHM) acquires a cosmological effect that fits the observed accelerating Hubble expansion for redshifts $z \leq 1$ (7.33 Gyr) accurately with only one free constant parameter. Conformal gravity (CG) has recently been fitted to anomalous rotation data for 138 galaxies. Conformal theory explains dark energy and does not require dark matter, providing a viable alternative to the standard $\Lambda \mathrm{CDM}$ paradigm. The theory precludes a massive Higgs particle but validates a composite gauge field $W_{2}$ with mass $125 \mathrm{GeV}$.
\end{abstract}

Keywords: conformal theory; $125 \mathrm{GeV}$ particle; dark matter; dark energy; dark galactic halo

PACS: 04.20.Cv; 98.80.-k; 11.15.-q

\section{Introduction}

Gravitational phenomena that cannot be explained by general relativity as formulated by Einstein are attributed to cold dark matter in the consensus $\Lambda C D M$ paradigm for cosmology. The search for tangible dark matter has continued without success for many years [1]. Dark energy $\Lambda$ remains without explanation.

Universal conformal symmetry offers an alternative paradigm, motivated by this situation. This requires local Weyl scaling covariance [2-4] for all massless elementary physical fields, without dark matter [5]. With no novel elementary fields, this extends conformal symmetry, valid for fermion and gauge boson fields [6], to both the metric tensor field of general relativity and the Higgs scalar field of elementary-particle theory $[7,8]$. This postulate implies conformal gravity CG [3,9-13] and the conformal Higgs model CHM [14-17].

The CHM determines model parameters that retain the Higgs mechanism but preclude a massive Higgs particle, replacing mass by implied dark energy in agreement with observed Hubble expansion [14,15], while validating a novel composite gauge field $W_{2}$ of mass $125 \mathrm{GeV}$ [18]. This paper reviews the logic of the CHM and its qualitative and quantitative justification by observed cosmological, gravitational, and particle physics data.

Conformal gravity (CG) [3] retains the logical structure of general relativity, replacing the Einstein-Hilbert Lagrangian density by a quadratic contraction of the conformal Weyl tensor [2]. CG ensures consistency of the gravitational field equations, while preserving subgalactic phenomenology $[3,9]$. The conformal Higgs model (CHM) introduces a gravitational term confirmed by observed accelerating Hubble expansion [3,14,15,17].

Substantial empirical support for this proposed break with convention is provided by recent applications of CG to galactic rotation velocities [19-24] and of the CHM to Hubble expansion [14,15], in a consistent model of extended dark galactic halos [16], without dark matter. Gravitational lensing by a galactic halo is attributed to the boundary condition of continuous gravitational acceleration across the outer boundary radius of the large spherical halo depleted of primordial matter fallen into the visible galaxy [16]. This accounts for the nonclassical acceleration parameter $\gamma$ deduced from anomalous orbital rotation velocities observed in the outer regions of galaxies [3]. As recently reviewed $[3,13,17]$, conformal theory fits observational data for an isolated galaxy without invoking dark matter, resolving 
several longstanding paradoxes. Higgs biquadratic parameter $\lambda$ is found to determine $W_{2}$ mass $125 \mathrm{GeV}$ [18]. Derivations of these results are summarized here.

\section{Depleted Halo Model}

CG and the CHM are consistent but interdependent [17] in the context of a depleted dark halo model [16] of an isolated galaxy. A galaxy of mass $M$ is modeled by spherically averaged mass density $\rho_{G} / c^{2}$ within $r_{G}$, formed by condensation of primordial uniform, isotropic matter of uniform mass density $\rho_{m} / c^{2}$ from a sphere of large radius $r_{H}$ [16]. The dark halo inferred from gravitational lensing and centripetal acceleration is identified with this depleted sphere [16]. A model valid for nonclassical gravitation can take advantage of spherical symmetry at large galactic radii, assuming classical gravitation within an effective galactic radius $r_{G}$. Non-spherical gravitation is neglected outside $r_{G}$. Given mean mass density $\bar{\rho}_{G} / c^{2}$ within $r_{G}$, this implies large empty halo radius $r_{H}=r_{G}\left(\bar{\rho}_{G} / \rho_{m}\right)^{\frac{1}{3}}$.

The unique Lagrangian density $\mathcal{L}_{g}$ of conformal gravity theory, constructed from the conformal Weyl tensor $[2,3,9]$, determines source-free Schwarzschild gravitational potential

$$
B(r)=-2 \beta / r+\alpha+\gamma r-\kappa r^{2},
$$

valid outside a spherically symmetric mass/energy source density $[9,12]$. This adds two constants of integration to the classical external potential: nonclassical radial acceleration $\gamma$ and halo cutoff parameter $\kappa[16]$.

The physically relevant particular solution for $B(r)$ [17] incorporates nonclassical radial acceleration $\gamma$ as a free parameter. Its value is determined by the halo model. Gravitational lensing by a spherical halo is observed as centripetal deflection of a photon geodesic passing into the empty halo sphere from the external intergalactic space with postulated universal isotropic mass/energy density $\rho_{m}$. The conformal Friedmann cosmic evolution equation implies dimensionless cosmic acceleration parameters $\Omega_{q}(\rho)$ [16] which are locally constant but differ across the halo boundary $r_{H}$. Smooth evolution of the cosmos implies observable particle acceleration $\gamma$ within $r_{H}$ proportional to $\Omega_{q}($ in $)-\Omega_{q}($ out $)=\Omega_{q}(0)-\Omega_{q}\left(\rho_{m}\right)$. Uniform cosmological $\rho_{m}$ implies constant $\gamma$ for $r \leq r_{H}$, independent of galactic mass [17]. This surprising result is consistent with recent observations of galactic rotational velocities for galaxies with directly measured mass $[25,26]$.

\section{Variational Field Equations}

Gravitational field equations are determined by metric functional derivative $X^{\mu \nu}=\frac{1}{\sqrt{-g}} \frac{\delta I}{\delta g_{\mu v}}$, where $g$ is the determinant of $g_{\mu v}$, and action integral $I=\int d^{4} x \sqrt{-g} \sum_{a} \mathcal{L}_{a}[3,27,28]$. Given $\delta \mathcal{L}=x^{\mu v} \delta g_{\mu v}, X^{\mu v}=x^{\mu v}+\frac{1}{2} \mathcal{L}^{\mu \nu}$. Scalar Lagrangian density $\mathcal{L}_{a}$ determines variational energy-momentum tensor $\Theta_{a}^{\mu \nu}=-2 X_{a}^{\mu \nu}$, evaluated for a solution of the field equations.

For fixed coordinates $x^{\mu}$, local Weyl scaling is defined by $g_{\mu \nu}(x) \rightarrow g_{\mu \nu}(x) \Omega^{2}(x)$ [2] for arbitrary real differentiable $\Omega(x)$. Conformal symmetry is defined by invariant action integral $I=\int d^{4} x \sqrt{-g} \mathcal{L}$. For any Riemannian tensor $T(x), T(x) \rightarrow \Omega^{d}(x) T(x)+\mathcal{R}(x)$ defines weight $d[T]$ and residue $\mathcal{R}[T]$. For a scalar field, $\Phi(x) \rightarrow \Phi(x) \Omega^{-1}(x)$, so that $d[\Phi]=-1$. Conformal Lagrangian density $\mathcal{L}$ must have weight $d[\mathcal{L}]=-4$ and residue $\mathcal{R}[\mathcal{L}]=0$ up to a 4-divergence [3]. For a bare conformal field, trace $g_{\mu \nu} X_{a}^{\mu v}=0$ [3]. Generalized Einstein equation $\sum_{a} X_{a}^{\mu \nu}=0$ is expressed as $X_{g}^{\mu v}=\frac{1}{2} \sum_{a \neq g} \Theta_{a}^{\mu \nu}$. Summed trace $\sum_{a} g_{\mu \nu} X_{a}^{\mu \nu}$ vanishes for exact field solutions.

The action integral defined by Lagrangian density $\mathcal{L}_{W}=-\alpha_{g} C_{\lambda}^{\mu \kappa v} C_{\mu \kappa \nu}^{\lambda}$ for Weyl tensor $C_{\lambda}^{\mu \kappa v}$, a traceless projection of the Riemann tensor [2], is conformally invariant. After removing a 4-divergence [3], $\mathcal{L}_{g}=-2 \alpha_{g}\left(R^{\mu v} R_{\mu v}-\frac{1}{3} R^{2}\right)$, where $R=g_{\mu v} R^{\mu v}$. Conformal symmetry fixes the relative coefficient of the two quadratic terms. For uniform mass/energy density $\bar{\rho}$ the Weyl tensor vanishes identically. Hence $X_{g}^{\mu v} \equiv 0$ for a uniform cosmos. 


\section{The Conformal Higgs Model}

Higgs $V\left(\Phi^{\dagger} \Phi\right)=-\left(w^{2}-\lambda \Phi^{\dagger} \Phi\right) \Phi^{\dagger} \Phi$ depends on two assumed constants $w^{2}$ and $\lambda[7,8]$. Nonzero $w^{2}$ and $\lambda$ are not determined by standard theory. The conformal Higgs model (CHM) introduces a gravitational term confirmed by observed Hubble expansion [14,15]. The CHM supports the Higgs mechanism, spontaneous SU(2) symmetry-breaking, which also breaks conformal symmetry [15] and invalidates a transformation connecting the two distinct metrics invoked for successful fits to galactic rotation and Hubble expansion [3].

In the conformal Higgs model, unique Lagrangian density $\mathcal{L}_{\Phi}[3,14,15]$ of Higgs scalar field $\Phi$ adds a gravitational term to $-V$, so that $\left(\partial_{\mu} \Phi\right)^{\dagger} \partial^{\mu} \Phi$ is augmented by

$$
\Delta \mathcal{L}_{\Phi}=\left(w^{2}-\frac{1}{6} R-\lambda \Phi^{\dagger} \Phi\right) \Phi^{\dagger} \Phi
$$

Scalar $R=g_{\mu v} R^{\mu v}$ is the trace of the Ricci tensor.

In uniform, isotropic geometry with uniform mass/energy density $\bar{\rho}, \mathcal{L}_{\Phi}$ implies a modified Friedmann equation $[5,14,29]$ for cosmic distance scale factor $a(t)$, with $a\left(t_{0}\right)=1$ at present time $t_{0}$ :

$$
\frac{\dot{a}^{2}}{a^{2}}+\frac{k}{a^{2}}-\frac{\ddot{a}}{a}=\frac{2}{3}\left(\bar{\Lambda}+\bar{\tau} c^{2} \bar{\rho}(t)\right) .
$$

$\bar{\Lambda}=\frac{3}{2} w^{2} \geq 0$ and $\bar{\tau} \sim-3 / \phi_{0}^{2} \leq 0$ are determined by parameters of the Higgs model [14].

By neglecting cosmic curvature $k$ in accordance with observational data, the sum rule $\Omega_{\Lambda}(t)+\Omega_{m}(t)+\Omega_{q}(t)=1$ follows if conformal Equation (3) is divided by $\dot{a}^{2} / a^{2}$, defining dimensionless Friedmann weights $\Omega_{\Lambda}=\frac{2}{3} \frac{\bar{\Lambda} a^{2}}{\dot{a}^{2}}, \Omega_{m}=\frac{2}{3} \frac{\bar{\tau} c^{2} \bar{\rho} a^{2}}{\dot{a}^{2}}$, and acceleration weight $\Omega_{q}=\frac{\ddot{a} a}{\dot{a}^{2}} \quad[14,15]$. Matter and radiation are combined in $\Omega_{m}$, while the Hubble function $H(t)=\frac{\dot{a}}{a}(t) \sim\left[T^{-1}\right]=h(t) H_{0}$ for Hubble constant $H_{0}=H\left(t_{0}\right)$. Setting $a\left(t_{0}\right)=1$, $h\left(t_{0}\right)=\frac{\dot{a}}{a}\left(t_{0}\right)=1$ in Hubble units of time $1 / H_{0}$, length $c / H_{0}$, and acceleration $H_{0}^{2} c / H_{0}=c H_{0}$. Evaluated at time $t$, Ricci scalar $R(t)=\frac{\ddot{a}}{a}+\frac{\dot{a}^{2}}{a^{2}}+\frac{k}{\dot{a}^{2}}$.

For $k=0$ the standard Friedmann equation, divided by $\frac{\dot{a}}{a}$, produces dimensionless sum rule $\Omega_{\Lambda}(t)+\Omega_{m}(t)=1$. $\Omega_{m}=1-\Omega_{\Lambda}$ requires mass density far greater than observed baryonic mass. This has been considered to be a strong argument for dark matter. Omitting $\Omega_{m}$ completely, with $k=0$, the conformal sum rule $\Omega_{\Lambda}(t)+\Omega_{q}(t)=1$ fits the observed data accurately for redshifts $z \leq 1(7.33 \mathrm{Gyr})[14,15,17]$. This eliminates any need for dark matter to explain Hubble expansion.

The luminosity distance $d_{L}(z)=(1+z) \chi(z)$, for $\Omega_{k}=0$, is shown in Table 1 for $\alpha=\Omega_{\Lambda}\left(t_{0}\right)=0.732$, where [15]

$$
\begin{array}{r}
\chi(z)=\int_{t(z)}^{t_{0}} \frac{d t}{a(t)}=\int_{z}^{0} d z(1+z) \frac{d t}{d z}= \\
\int_{0}^{z} \frac{d z}{\sqrt{2 \alpha \ln (1+z)+1}} .
\end{array}
$$

Observed redshifts have been fitted to an analytic function [30] with statistical accuracy comparable to the best standard $\Lambda \mathrm{CDM}$ fit, with $\Omega_{m}=0$. Table 1 compares $\mathrm{CHM} d_{L}(z)$ to this Mannheim function. As $\bar{\tau}$ is negative [3,14], cosmic acceleration $\Omega_{q}$ remains positive (centrifugal) back to the earliest time [14].

Conformal Friedmann Equation (3) [14,15] determines cosmic acceleration weight $\Omega_{q}$. With both weight parameters $\Omega_{k}$ and $\Omega_{m}$ set to zero, Equation (3) fits scaled Hubble function $h(t)=H(t) / H_{0}$ for redshifts $z \leq 1$, Table 1 , as accurately as standard LCDM, with only one free constant. This determines Friedmann weights, at present time $t_{0}$, $\Omega_{\Lambda}=0.732, \Omega_{q}=0.268$ [14]. Hubble constant $H\left(t_{0}\right)=H_{0}=2.197 \times 10^{-18} / \mathrm{s}$ [31] is independent of these data. The dimensionless sum rule [14] with $\Omega_{k}=0$ determines 
$\Omega_{q}\left(\rho_{m}\right)=1-\Omega_{\Lambda}-\Omega_{m}$ in the cosmic background, and $\Omega_{q}(0)=1-\Omega_{\Lambda}$ in the depleted halo [16].

Table 1. Scaled luminosity distance fit to Hubble data.

\begin{tabular}{lcccc}
\hline & & & Theory & Observed \\
$\mathbf{z}$ & $\boldsymbol{\Omega}_{\boldsymbol{\Lambda}}$ & $\boldsymbol{\Omega}_{\boldsymbol{q}}$ & $\boldsymbol{H}_{\mathbf{0}} \boldsymbol{d}_{\boldsymbol{L}} / \boldsymbol{c}$ Equation (4) & $\boldsymbol{H}_{\mathbf{0}} \boldsymbol{d}_{\boldsymbol{L}} / \boldsymbol{c}[30]$ \\
\hline 0.0 & 0.732 & 0.268 & 0.0000 & 0.0000 \\
0.2 & 0.578 & 0.422 & 0.2254 & 0.2265 \\
0.4 & 0.490 & 0.510 & 0.5013 & 0.5039 \\
0.6 & 0.434 & 0.566 & 0.8267 & 0.8297 \\
0.8 & 0.393 & 0.607 & 1.2003 & 1.2026 \\
1.0 & 0.363 & 0.637 & 1.6209 & 1.6216 \\
\hline
\end{tabular}

\section{Conformal Gravity}

The unique Lagrangian density $\mathcal{L}_{g}$ of conformal gravity, constructed from the conformal Weyl tensor $[2,3,9]$, determines Schwarzschild gravitational potential

$$
B(r)=-2 \beta / r+\alpha+\gamma r-\kappa r^{2},
$$

valid outside a spherically symmetric mass/energy source density $[9,12]$. Classical gravitation is retained at subgalactic distances by setting $\beta=\mathrm{Gm} / \mathrm{c}^{2}$ for a spherical source of mass $m$. CG adds two constants of integration to the classical external potential: nonclassical radial acceleration $\gamma$ and halo cutoff parameter $\kappa$ [16], with negligible effects at subgalactic distances [3].

From the CHM, observed nonclassical gravitational acceleration $\frac{1}{2} \gamma c^{2}$ in the halo is proportional to $\Delta \Omega_{q}=\Omega_{q}(0)-\Omega_{q}\left(\rho_{m}\right)=\Omega_{m}\left(\rho_{m}\right)$ [16], where, given $\rho_{m}$ and $H_{0}$, $\Omega_{m}\left(\rho_{m}\right)=\frac{2}{3} \frac{\bar{c} c^{2} \rho_{m}}{H_{0}^{2}}$ [14]. Thus, the depleted halo model determines constant $\gamma$ from uniform universal cosmic baryonic mass density $\rho_{m} / c^{2}$, which includes radiation energy density here.

Converted from Hubble units, this implies centripetal acceleration $\frac{1}{2} \gamma c^{2}=-c H_{0} \Omega_{m}\left(\rho_{m}\right)$ [16]. Positive $\rho_{m}$ implies $\Omega_{m}<0$ because coefficient $\bar{\tau}<0$ [3,14]. Hence $\Delta \Omega_{q}=\Omega_{m}<0$ is consistent with nonclassical centripetal acceleration $\frac{1}{2} \gamma c^{2}$, confirmed by inward deflection of photon geodesics observed in gravitational lensing [16]. This logic is equivalent to requiring radial acceleration to be continuous across the halo boundary $r_{H}$ :

$$
\frac{1}{2} \gamma_{H} c^{2}-c H_{0} \Omega_{q}(0)=-c H_{0} \Omega_{q}\left(\rho_{m}\right) .
$$

Signs here follow from the definition of $\Omega_{q}$ as centrifugal acceleration weight.

Given mass/energy source density $f(r)$ enclosed within $\bar{r}$, the field equation in the ES metric is $[9,12]$

$$
\partial_{r}^{4}(r B(r))=r f(r),
$$

for $f(r) \sim\left(\Theta_{0}^{0}-\Theta_{r}^{r}\right)_{m}$ determined by source energy-momentum tensor $\Theta_{m}^{\mu v}$ [3].

For constants related by $\alpha^{2}=1-6 \beta \gamma$ [12],

$$
y_{0}(r)=r B(r)=-2 \beta+\alpha r+\gamma r^{2}-\kappa r^{3},
$$

is a solution of the tensorial field equation for source-free $r \geq \bar{r}[9,12]$. Derivative functions $y_{i}(r)=\partial_{r}^{i}(r B(r)), 0 \leq i \leq 3$ satisfy differential equations

$$
\begin{aligned}
\partial_{r} y_{i}=y_{i+1}, 0 & \leq i \leq 2, \\
\partial_{r} y_{3} & =r f(r) .
\end{aligned}
$$


The general solution, for independent constants $c_{i}=y_{i}(0)$, determines coefficients $\beta, \alpha, \gamma, \kappa$ such that at endpoint $\bar{r}$

$$
\begin{array}{r}
y_{0}(\bar{r})=-2 \beta+\alpha \bar{r}+\gamma \bar{r}^{2}-\kappa \bar{r}^{3}, \\
y_{1}(\bar{r})=\alpha+2 \gamma \bar{r}-3 \kappa \bar{r}^{2}, \\
y_{2}(\bar{r})=2 \gamma-6 \kappa \bar{r}, \\
y_{3}(\bar{r})=-6 \kappa .
\end{array}
$$

Gravitational potential $B(r)$ is required to be differentiable and free of singularities. $c_{0}=0$ prevents a singularity at the origin. Values of $c_{1}, c_{2}, c_{3}$ can be chosen to match outer boundary conditions $\alpha=1, \gamma=0, \kappa=0$ at $r=\bar{r}$. This determines parameter $\beta$. Specific values of $\gamma$ and $\kappa$, consistent with Hubble expansion and the observed galactic dark halo [14,16], are fitted by adjusting $c_{1}, c_{2}, c_{3}$, subject to $c_{0}=0, \alpha^{2}=1-6 \beta \gamma$.

A particular solution for $B(r)[9,12]$, assumed by subsequent authors, derives an integral for $\gamma$ that vanishes for residual source density $\hat{\rho}$. This is replaced here by an alternative solution for which $\gamma$ is a free parameter [17].

For a single spherical solar mass isolated in a galactic halo, mean internal mass density $\bar{\rho}_{\odot}$ within $r_{\odot}$ determines an exact solution of the conformal Higgs gravitational equation, giving internal acceleration $\Omega_{q}\left(\bar{\rho}_{\odot}\right)$.

Given $\gamma$ outside $r_{\odot}$, continuous acceleration across boundary $r_{\odot}$,

$$
\frac{1}{2} \gamma_{\odot, i n} c^{2}-c H_{0} \Omega_{q}\left(\bar{\rho}_{\odot}\right)=\frac{1}{2} \gamma c^{2}-c H_{0} \Omega_{q}(0),
$$

determines constant $\gamma_{\odot \text {,in }}$ valid inside $\gamma_{\odot} \cdot \gamma_{\odot, \text { in }}$ is determined by local mean source density $\bar{\rho}_{\odot} \cdot \gamma$ in the halo is not changed. Its value is a constant of integration that cannot vary in the source-free halo [16,17]. Hence, there is no way to determine a mass-dependent increment to $\gamma$. This replaces the usually assumed $\gamma=\gamma_{0}+N^{*} \gamma^{*}$ by $\gamma=\gamma_{H}$, determined at halo boundary $r_{H}$.

Anomalous rotation velocities for 138 galaxies have been fitted to Equation (1) using four assumed universal parameters $\beta^{*}, \gamma^{*}, \gamma_{0}, \kappa[3,13,21,24]$ such that $\beta=N^{*} \beta^{*}=$ $G M / c^{2}, \gamma=\gamma_{0}+N^{*} \gamma^{*} . N^{*}$ is galactic baryonic mass $M$ in solar mass units. Inferred parameter values [3,22],

$$
\begin{gathered}
\beta^{*}=1.475 \times 10^{3} \mathrm{~m}, \gamma_{0}=3.06 \times 10^{-28} / \mathrm{m}, \\
\gamma^{*}=5.42 \times 10^{-39} / \mathrm{m}, \kappa=9.54 \times 10^{-50} / \mathrm{m}^{2},
\end{gathered}
$$

fit conformal gravity to galactic rotation velocities. The depleted halo model removes the galactic mass dependence of nonclassical acceleration parameter $\gamma$. For our Milky Way galaxy, with $N^{*}=6.07 \times 10^{10}[24,32]$, implied $\gamma_{H}=\gamma_{0}+N^{*} \gamma^{*}=6.35 \times 10^{-28} / \mathrm{m}$. Parameter $\kappa$ provides a cutoff of modified radial acceleration at a large halo radius [17].

\section{Reconciliation of the Two Distinct Gravitational Models}

CG and CHM must be consistent for an isolated galaxy and its dark halo, observed by gravitational lensing. CG is valid for anomalous outer galactic rotation velocities in the static spherical Schwarzschild metric, solving a differential equation for Schwarzschild gravitational potential $B(r)[3,9]$. The CHM is valid for cosmic Hubble expansion in the uniform, isotropic FLRW metric, solving a differential equation for Friedmann scale factor $a(t)$ [14]. Concurrent validity is achieved by introducing a common hybrid metric. The two resulting gravitational equations are decoupled by separating mass/energy source density $\rho$ into uniform isotropic mean density $\bar{\rho}$ and residual $\hat{\rho}=\rho-\bar{\rho}$, which extends only to galactic radius $r_{G}$ and integrates to zero over the defining volume.

The conformally invariant action integral of conformal gravity is defined by a quadratic contraction of the Weyl tensor, simplified by removing a 4-divergence $[3,13]$. For uniform density $\bar{\rho}$ the Weyl tensor vanishes identically, so that $X_{g}^{\mu \nu} \equiv 0$ for a uniform, isotropic cosmos. 
Observed excessive galactic rotational velocities have been studied and parametrized using conformal Weyl Lagrangian density $\mathcal{L}_{g}[3,13]$. The generalized Einstein equation exactly cancels any vacuum energy density. Hubble expansion has been parametrized using conformal Higgs scalar field Lagrangian density $\mathcal{L}_{\Phi}$ [14].

Metric tensor $g_{\mu \nu}$ is determined by conformal field equations derived from $\mathcal{L}_{g}+\mathcal{L}_{\Phi}$ [16], driven by energy-momentum tensor $\Theta_{m}^{\mu v}$, where subscript $m$ refers to conventional matter and radiation. The gravitational field equation within halo radius $r_{H}$ is

$$
X_{g}^{\mu \nu}+X_{\Phi}^{\mu v}=\frac{1}{2} \Theta_{m}^{\mu \nu}
$$

Defining mean density $\bar{\rho}_{G}$ and residual density $\hat{\rho}_{G}=\rho_{G}-\bar{\rho}_{G}$, and assuming $\Theta_{m}^{\mu v}(\rho) \simeq$ $\Theta_{m}^{\mu \nu}(\bar{\rho})+\Theta_{m}^{\mu \nu}(\hat{\rho})$, solutions for $r \leq r_{G}$ of the two equations

$$
X_{g}^{\mu \nu}=\frac{1}{2} \Theta_{m}^{\mu v}\left(\hat{\rho}_{G}\right), X_{\Phi}^{\mu v}=\frac{1}{2} \Theta_{m}^{\mu v}\left(\bar{\rho}_{G}\right)
$$

decouple and imply a solution of the full equation. This removes any mean density source from the $X_{g}$ equation, leaving only the residual density, which integrates to zero over a closed volume and cancels vacuum energy.

The decoupled solutions require a composite hybrid metric such as [17]

$$
d s^{2}=-B(r) d t^{2}+a^{2}(t)\left(\frac{d r^{2}}{B(r)}+r^{2} d \omega^{2}\right) .
$$

Solutions in the two distinct primitive metrics are made consistent by fitting parameters to boundary conditions and setting cosmic curvature constant $k=0$, justified by currently observed data.

\section{Baryonic Tully-Fisher and Radial Acceleration Relations}

Static spherical geometry defines Schwarzschild potential $B(r)$. For a test particle in a stable exterior circular orbit with velocity $v$ the centripetal acceleration is $a=v^{2}(r) / r=$ $\frac{1}{2} B^{\prime}(r) c^{2}$. Newtonian $B(r)=1-2 \beta / r$, where $\beta=G M / c^{2}$, so that $a_{N}=\beta c^{2} / r^{2}=G M / r^{2}$.

CG adds nonclassical $\Delta a$ to $a_{N}$, so that orbital velocity squared is the sum of $v^{2}\left(a_{N} ; r\right)$ and $v^{2}(\Delta a ; r)$, which crosses with equal and opposite slope at some $r=r_{T F}$. This defines a flat range of $v(r)$ centered at stationary point $r_{T F}$, without constraining behavior at large $r$.

MOND $[1,33,34]$ modifies the Newtonian force law for accelerations below an empirical scale $a_{0}$. Using $y=a_{N} / a_{0}$ as independent variable, for assumed universal constant $a_{0} \simeq 10^{-10} \mathrm{~m} / \mathrm{s}^{2}$, MOND postulates an interpolation function $v(y)$ such that observed radial acceleration $a=f\left(a_{N}\right)=a_{N} v(y)$. A flat velocity range approached asymptotically requires $a^{2} \rightarrow a_{N} a_{0}$ as $a_{N} \rightarrow 0$. For $a_{N} \gg a_{0}, v \rightarrow 1$ and for $a_{N} \ll a_{0}, v^{2} \rightarrow 1 / y$. This implies asymptotic limit $a^{2} \rightarrow a_{0} a_{N}$ for small $a_{N}$, which translates into an asymptotically flat galactic velocity function $v(r)$ for large orbital radius $r$ [33]. For $a_{N} \ll a_{0}$, MOND $v^{4}=a^{2} r^{2} \rightarrow G M a_{0}$, the empirical baryonic Tully-Fisher relation [20,35-37].

In conformal gravity (CG), centripetal acceleration $a=v^{2} / r$ determines exterior orbital velocity $v^{2} / c^{2}=r a / c^{2}=\beta / r+\frac{1}{2} \gamma r-\kappa r^{2}$, compared with asymptotic $r a_{N} / c^{2}=\beta / r$. If the asymptotic Newtonian function is valid at $r$ and $2 \kappa r / \gamma$ can be neglected, the slope of $v^{2}(r)$ vanishes at $r_{T F}^{2}=2 \beta / \gamma$. This implies that $v^{4}\left(r_{T F}\right) / c^{4}=\left(\beta / r_{T F}+\frac{1}{2} \gamma r_{T F}\right)^{2}=2 \beta \gamma[19,20]$. This is the Tully-Fisher relation, exact at stationary point $r_{T F}$ of the $v(r)$ function. Given $\beta=G M / c^{2}, v^{4}=2 G M \gamma c^{2}$, for relatively constant $v(r)$ centered at $r_{T F}$.

McGaugh et al. [25] have recently shown for 153 disk galaxies that observed radial acceleration $a$ is effectively a universal function of the expected classical Newtonian acceleration $a_{N}$, computed for the observed baryonic distribution. Galactic mass is determined directly by observation, removing uncertainty due to adjustment of mass-to-light ratios for individual galaxies in earlier studies. The existence of such a universal correlation function, $a\left(a_{N}\right)=a_{N} v\left(a_{N} / a_{0}\right)$, is a basic postulate of MOND [33,34]. 
CG implies a similar correlation function if nonclassical parameter $\gamma$ is mass-independent [26]. Outside an assumed spherical source mass, conformal Schwarzschild potential $B(r)$ determines circular geodesics such that $v^{2} / c^{2}=r a / c^{2}=\frac{1}{2} r B^{\prime}(r)=\beta / r+\frac{1}{2} \gamma r-\kappa r^{2}$. The Kepler formula is $r a_{N} / c^{2}=\beta / r$. Well inside a galactic halo boundary, $2 \kappa r / \gamma$ can be neglected. This defines correlation function $a\left(a_{N}\right)=a_{N}+\Delta a$ if $\Delta a=\frac{1}{2} \gamma c^{2}$ is a universal constant [26], which is implied by the depleted halo model [16]. This requires reconsideration of the definition $\gamma=\gamma_{0}+N^{*} \gamma^{*}$ used in fitting rotation data for 138 galaxies to CG $[3,22]$. For comparison with CG for the Tully-Fisher relation, CG would agree with MOND $v^{4}=G M a_{0}$ if $a_{0}=2 \gamma c^{2}$ [19], for mass-independent $\gamma$. CG $\gamma=6.35 \times 10^{-28} / \mathrm{m}$ implies MOND $a_{0}=1.14 \times 10^{-10} \mathrm{~m} / \mathrm{s}^{2}$.

\section{Higgs Parameter $\lambda$}

The conformal scalar field equation, including parametrized $\Delta \mathcal{L}_{\Phi}$, is $[3,14,17]$

$$
\frac{1}{\sqrt{-g}} \partial_{\mu}\left(\sqrt{-g} \partial^{\mu} \Phi\right)=-\left(\frac{1}{6} R-w_{0}^{2}+2 \lambda \Phi^{\dagger} \Phi\right) \Phi .
$$

For $k=0, \frac{1}{6} R(t)=h^{2}(t)\left(1+\Omega_{q}(t)\right)>h^{2}(t) \Omega_{\Lambda}=w(t)^{2}$, where $h(t)=\dot{a} / a$ in Hubble units [14].

Ricci scalar $R$ introduces gravitational effects. Time-dependent $R(t)=6\left(\xi_{0}(t)+\xi_{1}(t)\right)$, where $\xi_{0}(t)=\frac{\ddot{a}}{a}$ and $\xi_{1}(t)=\frac{\dot{a}^{2}}{a^{2}}+\frac{k}{a^{2}}$ [14]. For $h(t)=\dot{a} / a$ and $k=0, \frac{1}{6} R(t)=h^{2}(t)(2-$ $\left.\left.\left.\Omega_{\Lambda}(t)-\Omega_{m}(t)\right)=h^{2}(t)\left(1+\Omega_{q}(t)\right)>h^{2}(t) \Omega_{\Lambda}(t)\right)\right)=w(t)^{2}$. Hence $\zeta(t)=\frac{1}{6} R(t)-$ $w(t)^{2}>0$.

Only real-valued solution $\phi(t)$ is relevant in uniform, isotropic geometry. The field equation is

$$
\frac{\ddot{\phi}}{\phi}+3 \frac{\dot{a}}{a} \frac{\dot{\phi}}{\phi}=-\left(\frac{1}{6} R(t)-w(t)^{2}+2 \lambda \phi^{2}\right)
$$

Omitting $R$ and assuming constant $\lambda>0$, Higgs solution $\phi_{0}^{2}=w_{0}^{2} / 2 \lambda$ [7] is exact. All time derivatives drop out. In the conformal scalar field equation, cosmological time dependence of Ricci scalar $R(t)$, determined by the CHM Friedmann cosmic evolution equation, introduces nonvanishing time derivatives. $w_{0}^{2}=\frac{\dot{\phi}^{2}}{\phi^{2}}$ from the Higgs field covariant derivative is consistent with constant $\lambda$. Time-dependent terms in the scalar field equation can be included in $w^{2}(t)=\frac{\dot{\phi}^{2}}{\phi^{2}}-\frac{\ddot{\phi}}{\phi}-3 h(t) \frac{\dot{\phi}}{\phi}$. For $\zeta(t)=\frac{1}{6} R(t)-w^{2}(t)>0, \phi^{2}(t)=-\zeta(t) / 2 \lambda$ is an exact solution of Equation (17). $\zeta>0$ for computed $R(t)$ [14] implies $\lambda<0$.

$\hbar \phi\left(t_{0}\right)=174 \mathrm{GeV}[38]=1.203 \times 10^{44} \hbar H_{0} . \quad$ In Hubble units, for $\Omega_{m}=0$, $\zeta\left(t_{0}\right)=2 \Omega_{q}\left(t_{0}\right)=0.536$. For empirical $\phi(t), \lambda(t)=\zeta /\left(-2 \phi^{2}\right)$. Given $\zeta\left(t_{0}\right)$ and $\phi\left(t_{0}\right)$, dimensionless $\lambda\left(t_{0}\right)=-0.185 \times 10^{-88}[17]$.

\section{9. $W_{2}$ Particle and $Z_{2}$ Resonance}

Conformal theory obtains empirical parameters that preserve the standard electroweak model, but preclude a massive Higgs particle [17]. The observed LHC $125 \mathrm{GeV}$ resonance [39-41] requires an alternative explanation.

A model Hamiltonian matrix can be defined [18] in which indices 0,1 refer, respectively, to bare neutral scalar states $W W=g_{\mu \nu} W_{-}^{\mu} W_{+}^{\nu}, Z Z=g_{\mu v} Z^{\mu *} Z^{v}$. The assumed diagonal elements are $H_{00}=2 m_{W}=160 \mathrm{GeV}, H_{11}=2 m_{Z}=182 \mathrm{GeV}$, for empirical masses $m_{W}$ and $m_{Z}$. Intermediate quark and lepton states define a large complementary matrix $\tilde{H}$ indexed by $i, j \neq 0,1$, with eigenvalues $\epsilon_{i}$, and off-diagonal elements $\tilde{A}_{i 0}, \tilde{A}_{i 1}$. $\tilde{H}$ determines energy-dependent increments in a $2 \times 2$ reduced matrix

$$
H_{a b}-\mu_{a b}=H_{a b}-\sum_{i \neq 0,1} \tilde{A}_{a i}^{+}\left(\epsilon_{i}-\epsilon\right)^{-1} \tilde{A}_{i b} .
$$


$H_{01}-\mu_{01}=\left(W W\left|H_{\text {red }}\right| \mathrm{ZZ}\right)$ corresponds to Feynman diagrams for quark and lepton exchange. The most massive and presumably most strongly coupled intermediate field that interacts directly would be tetraquark $T=t \bar{b} b \bar{t}$, whose mass is estimated as $\epsilon_{T}=350 \mathrm{GeV}$. The very strong interaction between bare fields $\mathrm{ZZ}$ and $\mathrm{WW}$ is assumed to be dominated by tetraquark exchange.

A simplified estimate of $W_{2}$ energy is obtained by restricting intermediate states to the three color-indexed tetraquark states $T=t \bar{b} b \bar{t}$, and assuming elements $\tilde{A}_{T 0}, \tilde{A}_{T 1}$ of equal magnitude $\alpha / \sqrt{3}$. For the reduced $2 \times 2$ matrix, matrix increments $\mu_{a b} \simeq \mu(\epsilon)=\frac{\alpha^{2}}{\epsilon_{T}-\epsilon}$ are all defined by a single parameter $\alpha^{2}$. Secular equation

$$
\left(2 m_{W}-\mu(\epsilon)-\epsilon\right)\left(2 m_{Z}-\mu(\epsilon)-\epsilon\right)=\mu^{2}(\epsilon)
$$

is to be solved for two eigenvalues $\epsilon=E_{0}, E_{1}$.

It is found that identifying the model diboson $W_{2}$ with the recently observed LHC $125 \mathrm{GeV}$ resonance [39-41] confirms the empirical value of Higgs parameter $\lambda$. In Higgs $V\left(\Phi^{\dagger} \Phi\right)=-\left(w^{2}-\lambda \Phi^{\dagger} \Phi\right) \Phi^{\dagger} \Phi$, coefficient $w^{2}$ results from dressing the bare massless Higgs scalar field by neutral gauge field $Z_{\mu}$, while coefficient $\lambda$ results from dressing by $W_{2}[17,18]$.

Setting $E_{0}=125 \mathrm{GeV}=0.8644 \times 10^{44} \hbar H_{0}$ for the $W_{2}$ state, dominated by the bare $W W$ field, determines parameters $\alpha^{2}=4878 \mathrm{GeV}^{2}, \mu\left(E_{0}\right)=21.68 \mathrm{GeV}$ and $\tan \theta_{x}=0.6138$. Using $\alpha^{2}$ determined by $E_{0}$, the present model predicts $E_{1}=173 \mathrm{GeV}$, with $\mu\left(E_{1}\right)=27.62 \mathrm{GeV}$. This higher eigenvalue is the energy of a resonance $Z_{2}$ dominated by the bare $Z Z$ field. $Z_{2}$ decay into bare $W W$, two free charged gauge bosons, is allowed by energy conservation, but not into bare $Z Z$. Composite field $W_{2}$ cannot decay spontaneously into either WW or ZZ.

Identifying $E_{0}$ with the observed $125 \mathrm{GeV}$ resonance, and using $g_{w}=0.6312$ and $g_{z}=0.7165$ with computed $\tan \theta_{x}=0.6138$, the implied value of Higgs parameter $\lambda=-\frac{1}{4} g_{w}^{2} g_{z}^{2} \sin 2 \theta_{x}\left(\frac{\dot{\phi}_{0}}{\phi_{0}}\right)^{2} \hbar^{2} / m_{W_{2}}^{2} c^{4}=-0.455 \times 10^{-88}$ is consistent with its empirical value, $\lambda \simeq-10^{-88}[17,18]$.

Funding: This research received no external funding.

Institutional Review Board Statement: Not applicable.

Informed Consent Statement: Not applicable.

Data Availability Statement: Not applicable.

Conflicts of Interest: The author declares no conflict of interest.

\section{References}

1. Sanders, R.H. The Dark Matter Problem; Cambridge University Press: New York, NY, USA, 2010.

2. Weyl, H. Reine infinitesimal geometric. Math. Zeit. 1918, 2, 384. [CrossRef]

3. Mannheim, P.D. Alternatives to dark matter and dark energy. Prog. Part Nucl. Phys. 2006, 56, 340-445. [CrossRef]

4. Mannheim, P.D. Schwarzschild limit of conformal gravity in the presence of macroscopic scalar fields. Phys. Rev. D 2007, 75, 124006. [CrossRef]

5. Nesbet, R.K. Conformal gravity: Dark matter and dark energy. Entropy 2013, 15, 162. [CrossRef]

6. DeWitt, B.S. Relativity, Groups, and Topology; DeWitt, C., DeWitt, B.S., Eds.; Gordon and Breach: New York, NY, USA, 1964.

7. Higgs, P.W. Broken symmetries and the masses of gauge bosons. Phys. Rev. Lett. 1964, 13, 508. [CrossRef]

8. Cottingham, W.N.; Greenwood, D.A. An Introduction to the Standard Model of Particle Physics; Cambridge University Press: New York, NY, USA, 1998.

9. Mannheim, P.D.; Kazanas, D. Exact vacuum solution to conformal Weyl gravity and galactic rotation curves. Astrophys. J. 1989, 342, 635. [CrossRef]

10. Mannheim, P.D.; Kazanas, D. Newtonian limit of conformal gravity and the lack of necessity of the second order Poisson equation. Gen. Rel. Grav. 1994, 342, 337. [CrossRef]

11. Mannheim, P.D. Conformal cosmology with no cosmological constant. Gen. Rel. Grav. 1990, 22, 289. [CrossRef]

12. Mannheim, P.D. Some exact solutions to conformal Weyl gravity. Ann. N. Y. Acad. Sci. 1991, 631, 194. [CrossRef]

13. Mannheim, P.D. Making the case for conformal gravity. Found. Phys. 2012, 42, 388. [CrossRef] 
14. Nesbet, R.K. Cosmological implications of conformal field theory. Mod. Phys. Lett. A 2011, 26, 893. [CrossRef]

15. Nesbet, R.K. Dark energy density predicted and explained. Europhys. Lett. 2019, 125, 19001. [CrossRef]

16. Nesbet, R.K. Dark galactic halos without dark matter. Europhys. Lett. 2015, 109, 59001. [CrossRef]

17. Nesbet, R.K. Conformal theory of gravitation and cosmology. Europhys. Lett. 2020, 131, 10002. [CrossRef]

18. Nesbet, R.K. Conformal Higgs model: Gauge fields can produce a 125GeV resonance. Mod. Phys. Lett. A 2021, $36,2150161$. [CrossRef]

19. Mannheim, P.D. Are galactic rotation curves really flat? Astrophys. J. 1997, 479, 659. [CrossRef]

20. O'Brien, J.G.; Chiarelli, T.L.; Mannheim, P.D. Universal properties of centripetal accelerations of spiral galaxies. Phys. Lett. B 2018, $782,433$.

21. Mannheim, P.D.; O’Brien, J.G. Impact of a global quadratic potential on galactic rotation curves. Phys. Rev. Lett. 2011, 106, 121101. [CrossRef] [PubMed]

22. Mannheim, P.D.; O'Brien, J.G. Fitting galactic rotation curves with conformal gravity and a global quadratic potential. Phys. Rev. D 2012, 85, 124020. [CrossRef]

23. O'Brien, J.G.; Mannheim, P.D. Fitting dwarf galaxy rotation curves with conformal gravity. MNRAS 2012, 421, 1273. [CrossRef]

24. O'Brien, J.G.; Moss, R.J. Rotation curve for the Milky Way galaxy in conformal gravity. J. Phys. Conf. 2015, 615, 012002. [CrossRef]

25. McGaugh, S.S.; Lelli, F.; Schombert, J.M. The radial acceleration relation in rotationally supported galaxies. Phys. Rev. Lett. 2016, 117, 201101. [CrossRef] [PubMed]

26. Nesbet, R.K. Theoretical implications of the galactic radial acceleration relation of McGaugh, Lelli, and Schombert. MNRAS 2018, 476, L69. [CrossRef]

27. Weinberg, S. Gravitation and Cosmology: Principles and Applications of the General Theory of Relativity; Wiley: New York, NY, USA, 1972

28. Nesbet, R.K. Variational Principles and Methods in Theoretical Physics and Chemistry; Cambridge University Press: New York, NY, USA, 2003.

29. Friedmann, A. On the possibility of the world with constant negative curvature. Z. Phys. 1922, 10, 377. [CrossRef]

30. Mannheim, P.D. How recent is cosmic acceleration? Int. J. Mod. Phys. 2003, 12, 893. [CrossRef]

31. Collab, P. Planck 2015 results. XIII, Cosmological parameters. Astron. Astrophys. 2016, 594, A13.

32. McGaugh, S.S. Milky Way mass models and MOND. Astrophys. J. 2008, 683, 137. [CrossRef]

33. Milgrom, M. A modification of the Newtonian dynamics: Implications for galaxies. Astrophys. J. 1983, 270, 371. [CrossRef]

34. Famaey, B.; McGaugh, S. Modified Newtonian Dynamics (MOND): Observational phenomenology and relativistic extensions. Living Rev. Relativ. 2012, 15, 10. [CrossRef] [PubMed]

35. Tully, R.B.; Fisher, J.R. A new method of determining distances to galaxies. Astron. Astrophys. 1977, 54, 661.

36. McGaugh, S.S. The baryonic Tully-Fisher relation of galaxies with extended rotation curves and the stellar mass of rotating galaxies. Astrophys. J. 2005, 632, 859. [CrossRef]

37. McGaugh, S.S. A novel test of the modified Newtonian dynamics with gas rich galaxies. Phys. Rev. Lett. 2011, 106, 121303. [CrossRef] [PubMed]

38. Amsler, C. Review of particle physics. Phys. Lett. B 2008, 667, 1-6. [CrossRef]

39. della Negra, M.; Jenni, P.; Virdee, T.S. Journey in the search for the Higgs boson: The ATLAS and CMS experiments at the LHC. Science 2012, 338, 1560. [CrossRef] [PubMed]

40. ATLAS Collaboration. Observation of a new particle in the search for the standard model Higgs boson with the ATLAS detector at the LHC. Phys. Lett. B 2012, 716, 1-29. [CrossRef]

41. CMS Collaboration. Observation of a new boson at a mass of $125 \mathrm{GeV}$ with the CMS experiment at the LHC. Phys. Lett. B 2012, 716, 30-61. [CrossRef] 